\title{
ORIGINAL ARTICLE IN-HOSPITAL AND SHORT-TERM OUTCOMES OF ENDOVASCULAR TREATMENT IN PATIENTS WITH ACUTE THROMBOEMBOLIC OCCLUSION OF THE SUPERIOR MESENTERIC ARTERY
}

\author{
Yusuf Can ${ }^{1}$, Ibrahim Kocayiğit ${ }^{1}$, Muhammed Necati Murat Aksoy ${ }^{1}$, Emrah Akın¹, \\ Muhammed Burak Kamburoglu' ${ }^{1}$, Harun Kılıc ${ }^{1}$, Ramazan Akdemir ${ }^{1}$ \\ ${ }^{1}$ Sakarya University, Turkey
}

\begin{abstract}
Objectives: To investigate the outcome of endovascular treatment in acute thromboembolic occlusion of the superior mesenteric artery.

Methodology: Eighteen consecutive patients who underwent endovascular treatment in acute thromboembolic occlusion of the superior mesenteric artery from January 2013 to September 2019 were included in the study. Thirty-day mortality, avoidance of laparotomy, angiographic success, length of hospital stay and complication rates were assessed.

Results: The patients were 46 to $87(70.5 \pm 13.0)$ year-old, and $9(50 \%)$ were male. In our study, 30-day mortality rate was $33.3 \%$. The remaining patients were discharged uneventfully. Our complete and partial success rates was $61.1 \%$ and $38.9 \%$ respectively on angiographic assessment. The mean length of hospital stay was $7.8 \pm 5.0$ days in the remaining patients. Short bowel syndrome, respiratory failure requiring mechanical ventilation support or renal failure requiring dialysis were not observed in remaining patients. Laparotomy was needed for eight patients after endovascular treatment.

Conclusion: The data showed that endovascular treatment is a technically feasible, effective and reliable alternative treatment method in a selected group of patients with acute thromboembolic occlusion of the superior mesenteric artery.

Keywords: Thromboembolic occlusion, superior mesenteric artery, endovascular treatment

Citation: Can Y, Kocayiğit I, Aksoy MNM, Akın E, Kamburoglu MB, Kılıc H, Akdemir R. In-Hospital and ShortTerm Outcomes of Endovascular Treatment in Patients with Acute Thromboembolic Occlusion of the Superior Mesenteric Artery. Pak Heart J. 2021;54(02):186-190. DOI: https://doi.org/10.47144/phj.v54i2.2097
\end{abstract}

\section{INTRODUCTION}

Acute mesenteric ischemia is a life-threatening disease, resulting in ischemia, cellular damage and necrosis as a result of sudden cessation of the intestines. Although it is rarely, in-hospital mortality is still very high and could reach up to 93\%. ${ }^{1}$ The most important reason for this condition that there is no obvious specific symptoms and signs, so the diagnosis delay. Most of the acute mesenteric ischemia is caused by mesenteric arterial embolism (50\%) and mesenteric arterial thrombosis (15-25\%). ${ }^{2,3}$ The embolus typically incline to locate at points of normal anatomic narrowing. Because superior mesenteric artery (SMA) has a large diameter and low take-off angle from the aorta, it is most affected. The majority of emboli is observed 3 to $10 \mathrm{~cm}$ distal to the origin of the SMA. Thrombi usually occurs at the origin of SMA. Thrombosis of the SMA is usually linked to pre-existing chronic atherosclerotic disease leading to stenosis. Treatment options include thrombolytic therapy, endovascular, surgery and combinations of these treatments. To our knowledge, there are no randomised clinical studies on those with acute thromboembolic occlusion of the SMA (ATOS), the optimal treatment strategy is not fully known, and endovascular treatment could be an alternative treatment method due to the risk of high surgical mortality. ${ }^{4}$ In the last two decades, endovascular treatments have been increasingly used with technological developments.5 Herein, we assessed the outcome of endovascular treatment in acute thromboembolic occlusion of the SMA.

\section{METHODOLOGY}

This was a single-center retrospective study carried out between January 2013 and September 2019 at the Sakarya University Faculty of Medicine. Patients included were those who underwent endovascular intervention due to acute thromboembolic occlusion of the SMA and patients with abdominal pain and $50 \%$ or more stenosis due to thromboembolism in SMA. Patients excluded were with non-occlusive mesenteric ischemia, ischemia due to aortic dissection and mesenteric venous thrombosis. Technical success of the endovascular revascularization procedure was determined as the residual stenosis of the formerly occluded artery less than $30 \%$ in diameter and without migration of small thromboemboli towards branches, along with rapid flow and visible contrast 
reaching the whole bowel. Partial success was determined as reestablished or improved flow of contrast to the corresponding bowel either with residual luminal caliber more than $30 \%$ or as occurrence of small thromboemboli migration towards distal vessels. ${ }^{6,7}$ The diagnosis of all patients was confirmed by computerized tomography (CT) angiography and digital substraction angioraphy (DSA). A number of features show the bowel wall necrosis and perforation, including pneumatosis intestinalis, pneumoperitoneum, submucosal hemorrhage, variable amounts of free fluid, bowel wall thickness. All patients or next of kin were informed about percutaneous procedures and informed consent was obtained. Ethics committee approval was received for this study from the Sakarya University Faculty of Medicine (Approval Date: 15.09.2019; Approval Number: 71522473/050.01.04/294)

The primary outcome was mortality within 30 days. The secondary endpoints included angiographic success, avoidance of laparotomy, complication rates, and length of hospital stay. The patients were evaluated at the 1st, 6th and 12th months of the procedure and additional imaging was performed for patients with gastrointestinal complaints.

After an intravenous (IV) heparin bolus of 5000 units, a continuous infusion of heparin at 12-15 units $/ \mathrm{kg} / \mathrm{hr}$ is ordered. Patients were treated with aspirin, clopidogrel, vitamin $\mathrm{K}$ antagonist alone or combination therapy with these drugs according to arrhythmia, embolism and thrombus. A 6 Fr sheath was inserted in the right femoral artery under local anaesthesia and a 6 Fr right Judkins guiding catheter was used to cannulate the SMA ostium. The SMA was evaluated with at least two images, including lateral and anterior views for arterial anatomy and stenosis / occlusion. Narrowing of $50 \%$ and over was evaluated as stenosis and $100 \%$ stenosis as occlusion with DSA. The degree of SMA-stenosis was evaluated using visual estimation. 8-Fr sheat was inserted into the femoral artery after the culprit lesion was identified. Then, the 7-Fr / 8-Fr renal double curved (RDC) or JR-4 guiding catheter was used to engage the ostium of the SMA. A 5/6 French guiding catheter was advanced through a 7/8 french catheter. Manual thrombus aspiration was performed with a 50-cc syringe. Balloon angioplasty with various sizes $(4.0 \times 20 \mathrm{~mm}, 4.5 \mathrm{x}$ $20 \mathrm{~mm}$ or $5.0 \times 20 \mathrm{~mm}$ balloons) was performed when thrombus aspiration failed. After balloon angioplasty, a stent was implanted when existence of dissection or atherosclerotic plaque with a residual stenosis of $>50 \%$. Self-expandable stents were implanted in patients with dissection after balloon angioplasty or balloon expandable stents were implanted in patients with ostial/proximal atherosclerotic lesions. Patients without atrial fibrilation were discharged one day after stent implantation on dual antiplatelet therapy with 100 $\mathrm{mg}$ of aspirin and $75 \mathrm{mg}$ of clopidogrel daily for one month followed by single antiplatelet therapy. Patients with atrial fibrilation were discharged on $75 \mathrm{mg}$ of clopidogrel daily and vitamin $\mathrm{K}$ antagonist for one month followed by single anticogulant therapy.

Data were analysed using SPSS 17.0 (SPSS, Inc., Chicago, IL, USA). Compliance of the variables with the normal distribution was determined using the Kolmogorov-Smirnov test. Continuous variables showing a normal distribution were specified as mean \pm standard deviation, whereas those showing no normal distribution were specified as the median (the smallest and greatest values). Categorical data were expressed in percentages.

\section{RESULTS}

A total of 18 patients (9 males) undergoing initial endovascular intervention due to acute thromboembolic occlusion of the SMA were included in the study. Average patient age was 70.5 \pm 13.0 years. The basic features of the patients are shown in Table 1 and Table 2. From the 18 patients with acute SMA stenosis/occlusion; 14 patients had occlusion, and 4 patients had stenosis. According to the etiology, 15 patients had embolism in the main trunk of SMA and 3 patients had ostial stenosis/occlusion due to thrombosis. Twelve of the patients with embolism had atrial fibrillation. Four of these patients were under anticoagulant therapy. Endovascular treatment was performed in 18 patients due to the lack of definite or suspected necrosis. In addition to peritonitis findings, necrosis was observed in 8 (\% 44.4) patients and necrosis was confirmed by laparotomy after endovascular treatment and these patients underwent surgery (Patients 1, 2, 7, 8, 12, 14, 15, and 18). The interval from symptom onset to treatment was $13.6 \pm 7.1$ hours, less than 12 hours in 10 cases while more than 12 hours in 8 cases. None of the patients underwent thrombolytic therapy due to the risk of intestinal bleeding. Although balloon angioplasty and thrombus aspiration was performed in all patients, stent implantation was performed in 5 patients (Patients 6, 8, 10, 11, 17). Stent was implanted in two patients after balloon angioplasty due to the dissection (Patients 6 and 8) and in three patients because of osteal lesion and $50 \%$ stenosis after balloon angioplasty (Patients 10, 11, 17). Partial success was achieved in 7 patients and 
complete success was achieved in 11 patients. The mortality rate was $35 \%$ in patients with partial success, and $65 \%$ in patients with complete success. Six patients who were diagnosed too late and underwent bowel resection died due to sepsis, hemorrhage and multiorgan failure after endovascular treatment (Patients 2, 8, 12, 14, 15, and 18). The mean length of hospital stay was 7.8
+/- 5.0 days in the remaining patients. The remaining patients were discharged uneventfully. Respiratory failure requiring mechanical ventilation support or renal failure requiring dialysis were not observed. No short bowel syndrome was observed in remaining patients. The in-hospital mortality rate was $33.3 \%$. No death was observed in the onemonth follow-up in remaining patients.

Table 1: Demographic and clinical features of patients with acute thromboembolic occlusion superior mesenteric artery

\begin{tabular}{|c|c|c|c|c|c|}
\hline Patient No & Age/Sex & Time of treatment & Lesion/Type & $\begin{array}{l}\text { Risk Factors } \\
\end{array}$ & Stenosis degree \\
\hline 1 & $69 / \mathrm{F}$ & $15 \mathrm{H}$ & Main trunk/ Emboli & $\mathrm{DM}, \mathrm{HT}, \mathrm{AF}$ & SMA: $100 \%$ \\
\hline 2 & $70 / \mathrm{M}$ & $28 \mathrm{H}$ & Main trunk/ Emboli & $\mathrm{HT}, \mathrm{AF}$ & SMA: $100 \%$ \\
\hline 3 & $79 / \mathrm{F}$ & $11 \mathrm{H}$ & Main trunk/ Emboli & DM, HT, AF, CAD & SMA: $100 \%$ \\
\hline 4 & $81 / \mathrm{M}$ & $7 \mathrm{H}$ & Main trunk/ Emboli & HT & SMA: $100 \%$ \\
\hline 5 & $67 / M$ & $9 \mathrm{H}$ & Main trunk/ Emboli & HT, DM, AF & SMA: $100 \%$ \\
\hline 6 & $46 / \mathrm{M}$ & $5 \mathrm{H}$ & Main trunk/ Emboli & HT, DM, CAD & SMA: $100 \%$ \\
\hline 7 & $74 / \mathrm{F}$ & $16 \mathrm{H}$ & Main trunk/ Emboli & $\mathrm{DM}, \mathrm{AF}$ & SMA: $100 \%$ \\
\hline 8 & $87 / \mathrm{F}$ & $30 \mathrm{H}$ & Main trunk/ Emboli & HT, AF & SMA: $100 \%$ \\
\hline 9 & $62 / \mathrm{M}$ & $11 \mathrm{H}$ & Main trunk/ Emboli & HT, DM, AF & SMA: $100 \%$ \\
\hline 10 & $70 / \mathrm{M}$ & $10 \mathrm{H}$ & Osteal/Thrombi & $\mathrm{DM}, \mathrm{HT}$ & SMA:90\% \\
\hline 11 & 77/M & $9 \mathrm{H}$ & Osteal/Thrombi & HT, CAD & SMA:90\% \\
\hline 12 & $85 / \mathrm{F}$ & $14 \mathrm{H}$ & Main trunk/ Emboli & $\mathrm{HT}, \mathrm{CAD}, \mathrm{AF}$ & SMA: $100 \%$ \\
\hline 13 & $87 / \mathrm{M}$ & $7 \mathrm{H}$ & Main trunk/ Emboli & HT, CAD, DM, AF & SMA:90\% \\
\hline 14 & $77 / \mathrm{M}$ & $16 \mathrm{H}$ & Main trunk/ Emboli & $\mathrm{AF}, \mathrm{HT}, \mathrm{DM}$ & SMA: $100 \%$ \\
\hline 15 & $49 / \mathrm{F}$ & $21 \mathrm{H}$ & Main trunk/ Emboli & HT & SMA: $100 \%$ \\
\hline 16 & $81 / \mathrm{F}$ & $9 \mathrm{H}$ & Main trunk/ Emboli & $\mathrm{HT}, \mathrm{AF}$ & SMA: $100 \%$ \\
\hline 17 & $57 / \mathrm{F}$ & $8 \mathrm{H}$ & Osteal/Thrombi & HT, AF & SMA:90\% \\
\hline 18 & $51 / \mathrm{F}$ & $18 \mathrm{H}$ & Main trunk/ Emboli & & SMA: $100 \%$ \\
\hline
\end{tabular}

AF: Atrial Fibrillation, CAD: Coronary Artery Disease, DM: Diabetes Mellitus, F: Female, H: Hour, HT: Hypertension, M: Male, SMA: Superior Mesenteric Artery

Table 2: Management and prognosis of patients with acute thromboembolic occlusion superior mesenteric artery

\begin{tabular}{|c|c|c|c|c|c|}
\hline Patient No & Treatment & Technical Success & Hospitalization Duration & Follow-up & 30-days Survival \\
\hline 1 & TA, BA & Complete & $9 \mathrm{D}$ & $22 \mathrm{M}$ & Yes \\
\hline 2 & TA, BA & Partial & NA & NA & No \\
\hline 3 & TA, BA & Partial & $12 \mathrm{D}$ & $49 \mathrm{M}$ & Yes \\
\hline 4 & TA, BA & Complete & $5 \mathrm{D}$ & $40 \mathrm{M}$ & Yes \\
\hline 5 & TA, BA & Complete & $7 \mathrm{D}$ & $56 \mathrm{M}$ & Yes \\
\hline 6 & TA, BA, Stent & Complete & $3 \mathrm{D}$ & $45 \mathrm{M}$ & Yes \\
\hline 7 & TA, BA & Partial & $20 \mathrm{D}$ & NA & No \\
\hline 8 & TA, BA, Stent & Partial & NA & $49 \mathrm{M}$ & Yes \\
\hline 9 & TA, BA & Complete & $6 \mathrm{D}$ & Yes \\
\hline 10 & TA, BA, Stent & Complete & $13 \mathrm{D}$ & $26 \mathrm{M}$ & Yes \\
\hline 11 & TA, BA, Stent & Complete & $5 \mathrm{D}$ & NA & No \\
\hline 12 & TA, BA & Partial & NA & NA & Yes \\
\hline 13 & TA, BA & Complete & $4 \mathrm{D}$ & No \\
\hline 14 & TA, BA & Partial & NA & NA & No \\
\hline 15 & TA, BA & Complete & NA & $4 \mathrm{M}$ & Yes \\
\hline 16 & TA, BA & Complete & $6 \mathrm{D}$ & Yes \\
\hline 17 & TA, BA, Stent & Complete & 3 D & No \\
\hline 18 & TA, BA & Partial & NA & NA & \\
\hline
\end{tabular}

BA:Balloon Angioplasty, D: Day, M: Month, NA:Not Available, TA: Thrombus Aspiration

\section{DISCUSSION}

Because ATOS has a similar symptoms like other acute abdominal conditions, the diagnosis of ATOS is often delayed. As soon as the diagnosis of ATOS is suspected, CT angiography should be performed immediately. Besides CT angiography can exclude other acute abdominal conditions, it shows ischemia/necrosis and contrast enhancement in SMA. Acute mesenteric ischemia should be kept in mind especially in patients with atrial fibrillation, congestive heart failure, hypercoagulopathy, cardiovascular disease and risk factors for cardiovascular disease. 
In most patients, the source of embolism is left atrium, left ventricle and valvular disease. ${ }^{8}$ Three of our patients had thrombosis with atherosclerotic plaque in SMA ostium. 15 patients had embolic etiology and 12 of them had atrial fibrillation.

The initial treatment of the patients without peritonitis and necrosis is unclear and controversial. We performed endovascular intervention as initial treatment. These patients, who may benefit from endovascular treatment without laparotomy, can be identified by taking into account the time, physical examination findings, new laboratory findings, new biomarkers, new imaging techniques or new findings of CT angiography.

The treatment modality varies depending on whether there are signs of peritonitis and necrosis. Due to the lack of prospective studies and the low number of patients in the retrospective studies, it is controversial whether endovascular techniques or endovascular treatment will be the optimal treatment with the development of endovascular techniques. In recent years, endovascular treatment has been applied as an alternative method for surgery in patients without necrosis findings without signs of peritonitis, but laparotomy initial treatment is applied in patients with definite signs of peritonitis and necrosis findings. ${ }^{5}$ Recently, techniques such as thrombolytic, percutaneous endovascular therapies and retrograde open mesenteric stenting can be used instead of surgical treatment. ${ }^{9-11}$ Previous studies have shown that endovascular treatment results in less bowel resection, less laparotomy, less mortality, fewer hospital stays, and less renal failure, respiratory failure compared to surgery. ${ }^{12}$ Arthurs et al. reported that, compared with traditional open revascularization therapy, endovascular treatment can result in significantly lower in-hospital mortality, lower laparotomy rates, a significantly decreased rate of bowel resection during surgical exploration, and fewer complications as a result of respiratory or renal failure. Arthur and colleagues reported $87 \%$ of technical success and $69 \%$ of laparotomy and $36 \%$ mortality rate with endovascular treatment. ${ }^{12}$ In another study, it was shown that technical success was $91.9 \%$, laparotomy $73 \%$ and in-hospital mortality $27 \%$, respectively. ${ }^{13}$ In our study, 30-day mortality and laparotomy rate were $33.3 \%$ and $44.4 \%$, respectively. Our complete success and partial success rates was $61.1 \%$ and $38.9 \%$ respectively. In our study; primary outcomes and technical success rates are similar when compared with other studies. Compared to previous studies, we have seen lower incidence of need of surgery.

Percutaneous techniques include manual thrombus aspiration, balloon angioplasty, stenting, mechanical thrombectomy. ${ }^{5,14}$ While thrombus aspiration and balloon angioplasty may be mostly sufficient in the lesions of the SMA main trunk, they are not sufficient enough in the osteal lesions. Three of the patients with stent implantation had osteal lesions.

Although thrombolytic provides a good result in early period, it is not recommended because of the risk of bleeding after developing intestinal necrosis. ${ }^{15,16}$ Catheter-directed thrombolysis may cause necrosis due to prolonged duration. Even partial successful revascularization may be sufficient in mesenteric interventions because collateral circulation between celiac truncus, inferior mesenteric artery, drummond artery, marginal artery, distal arcade collaterals provides adequate perfusions of distal vascular segments. Therefore, we did not apply thrombolytic treatment to any of our patients. We also think that thrombolytic therapy may cause unpredictable bleedings, which may increase mortality.

Early diagnosis and treatment is the most important predictor of mortality. The duration of resistance to ischemia may be related to atherosclerosis, collateral and location of the stenosis/occlusion. One of the most important indicators of mortality is the time from symptom onset to treatment. If this period is more than 12 hours, the rate of the mortality increases. Revesz et al. showed that patients who received treatment in less than 12 hours had a lower mortality than patients who received treatment for more than 12 hours. ${ }^{17}$ Eight of our patients were treated for more than 12 hours. We observed less mortality, less hospitalization, less laparotomy in patients who came in less than 12 hours.

Two types of percutaneous embolectomy are performed. The first is manual aspiration embolectomy with a large diameter catheter. ${ }^{5}$ The other is mechanical thrombectomy with hydrodynamic devices. For this purpose, successful results were obtained with rotarex, aspirex, and angiojet system. ${ }^{18-20}$ Because we did not have hydrodynamic devices in our hospital, we performed manual aspiration embolectomy with a large diameter catheter in all patients.

The limitations of our study were the small number of patients, single-centered, retrospective and no comparison with surgery.

\section{CONCLUSION}

As a result, percutaneous endovascular treatment in acute SMA stenosis / occlusion is an effective and reliable method especially in patients without intestinal necrosis. The results of endovascular treatments including balloon angioplasty and thromboaspiration are quite promising, especially in patients presenting in the early stages of the disease. These results should be supported by randomized controlled trials. Also, randomized trials are needed to thoroughly investigate the benefits and risks of 
endovascular treatment of ATOS, in comparison to a surgical approach.

\section{AUTHORS' CONTRIBUTION}

YC: Concept and design, data acquisition, interpretation, drafting, final approval, and agree to be accountable for all aspects of the work. IK, MNMA, EA, MBK, HK, RA: Data acquisition, interpretation, drafting, final approval and agree to be accountable for all aspects of the work.

Conflict of interest: Authors declared no conflict of interest.

\section{REFERENCES}

1. Schoots IG, Koffeman GI, Legemate DA, Levi M, van Gulik TM. Systematic review of survival after acute mesenteric ischaemia according to disease aetiology. Br J Surg. 2004;91:17-27.

2. Acosta S. Mesenteric ischemia. Curr Opin Crit Care 2015;21:171-8.

3. Clair DG, Beach JM. Mesenteric Ischemia. N Engl J Med. 2016;374:959-68

4. Block TA. Acosta S. Bjorck M. Endovascular and open surgery for acute occlusion of the superior mesenteric artery. Journal of Vasc ular Surgery. 2010;52:959-66.

5. Chen TY, Wu CH, Hsu WF, Lin L, Wang RH, Lai CL, et al. Primary Endovascular Intervention for Acute Mesenteric Ischemia Performed by Interventional Cardiologists -A Single Center Experience. Acta Cardiol Sin. 2017;33:439-46

6. Funaki B. Microcatheter embolization of lower gastrointestinal hemorrhage:an old idea whose time has come. Cardiovasc Intervent Radiol 2004;27:591-9.

7. Kuo WT, Lee DE, Saad WE, Patel N, Sahler LG, Waldman DL. Superselective microcoil embolization for the treatment of lower gastrointestinal hemorrhage. J Vasc Interv Radiol. 2003;14:15039.

8. Stanley JC. Mesenteric arterial occlusive and aneurysmal disease. Cardiol Clin. 2002;20:611-22.
9. Schoots IG, Levi MM, Reekers JA, Lameris JS, van Gulik TM. Thrombolytic therapy for acute superior mesenteric artery occlusion. J Vasc Interv Radiol. 2005;16:317-29.

10. Brountzos E. Emergency endovascular treatment of a superior mesenteric artery occlusion. Cardiovasc Intervent Radiol. 2001;24:57-60

11. Wyers MC. Powell RJ. Nolan BW. Cronenwett JL. Retrograde mesenteric stenting during laparotomy for acute occlusive mesenteric ischemia. J Vasc Surg. 2007;45:269-75.

12. Arthurs ZM. Titus J. Bannazadeh M. Eagleton MJ. Srivastava S. Sarac TP, et al. A comparison of endovascular revascularization with traditional therapy for the treatment of acute mesenteric ischemia. J Vasc Surg. 2011;53:698-704.

13. Raupach J, Lojik M, Chovanec V, Renc O, Strýček M, Dvořák P, et al. Endovascular management of acute embolic occlusion of the superior mesenteric artery: a 12-year single-centre experience. Cardiovasc Intervent Radiol. 2016;39:195-203.

14. El Farargy M, Abdel Hadi A, Abou Eisha M, Bashaeb K, Antoniou GA. Systematic review and meta-analysis of endovascular treatment for acute mesenteric ischaemia. Vascular. 2017;25:430-438.

15. Miyazawa T, Ueki K, Wakakuwa R. A case of acute superior mesenteric artery occlusion treated by intraarterial infusion of urokinase and prostaglandin E1. J Jpn Surg Assoc. 2004; 65:32936.

16. Acosta S. Sonesson B. Resch T. Endovascular therapeutic approaches for acute superior mesenteric artery occlusion. Cardiovasc Intervent Radiol. 2009;32:896-905.

17. Szabóné Révész E. Acute mesenteric ischemia: analysis of cases admitted to a hospital during 10 years (2001-2010). Orv Hetil. 2012;153(36):1424-32.

18. Zhang Z. Chen X. Zhu R. Percutaneous Mechanical Thrombectomy Treatment of Acute Superior Mesenteric Artery Embolism. EJVES Short Rep. 2017;34:17-20.

19. Kuhelj D. Kavcic P. Popovic P. Percutaneous mechanical thrombectomy of superior mesenteric artery embolism. Radiol Oncol. 2013;47:239-243.

20. Ballehaninna UK. Hingorani A. Ascher E. Shiferson A. Marks N. Aboian E. et al. Acute superior mesenteric artery embolism: reperfusion with AngioJet hydrodynamic suction thrombectomy and pharmacologic thrombolysis with the EKOS catheter. Vascular. 2012;20:166-9.

\section{Address for Correspondence:}

Dr. Yusuf Can, Department of Cardiology, Sakarya University, Turkey.

Email: dr.ycan@hotmail.com 